\title{
Rehearsal, generative processes, and the activation of underlying stimulus representations
}

\author{
JOHN G. SEAMON \\ Wesleyan University, Middletown, Connecticut 06457
}

\begin{abstract}
Previous research found that subjects engaged in mental rehearsal could reduce stimulus encoding time in classification and identification tasks by generating an appropriate representation of the probe stimulus prior to its presentation. The present experiment examined the types of representational codes activated by generative processes in character classification. Numerical stimuli were employed which could differ visually, yet have the same name and meaning (e.g., TWO, 2, II, \& . ). The results indicate that generative processes may activate visual and nonvisual memory codes prior to the encoding of an external stimulus. Effects of generative processes were discussed in terms of an activation model which defines the function of rehearsal in perceptual processing. Attention, through rehearsal, activates stimulus representations which are described by distributions of activation which change continuously over time. The momentary state of each distribution determines the rate of stimulus encoding.
\end{abstract}

The quickness with which an impression is perceived is proportional to the concentration of expectation, of voluntary attention upon it (Külpe, 1895, p. 432).

The study of the role of conscious attention in human performance is at least as old as the history of psychology. Effects of expectancy on reaction time were observed by Hartmann in his studies of the sources of error in the astronomer's personal equation as long ago as 1858 (Boring, 1950). By the 1860 s and $1870 \mathrm{~s}$, it was evident that expectancy, preparation, and attention were important determiners of performance. Reference to this firm belief can be found in many of the early texts of experimental psychology (e.g., James, 1890; Külpe, 1895; Titchener, 1908). Pachella (1975) notes that continued interest in this topic is evident by the fact that experimental contributions have been made in each decade of psychology's history (see Egeth \& Bevan, 1973, for a review).

In this tradition, a series of recent experiments on

This research was supported by NIH Grant 1-RO3-MH-27126-01 and a small Wesleyan University grant-in-aid of research. The paper was written while the author was on a half-year sabbatical in the Mathematical Psychology Laboratory of Rockefeller University. I would like to express my appreciation to William $\mathrm{K}$. Estes and the people in the Math Psych Lab, including Edward E. Smith and Douglas Medin; their company made a brief visit pass too quickly. Appreciation is also expressed to Sylvan Kornblum, Alfred Kristofferson, and Michael Posner for constructive comments. Requests for reprints should be sent to John G. Seamon, Department of Psychology, Wesleyan University, Middletown, Connecticut 06457. character classification and identification has shown that probe encoding time is a variable that can be influenced by a generative processing mechanism (Seamon, 1976a, 1976b; Seamon \& Wright, 1976). After rehearsing an array of target letters at a selfpaced or externally paced rate, subjects either vocally identified or manually classified a probe item in terms of target membership. The results have shown identification and classification times to be faster when the probe is the same as the last rehearsed item than when it is different. This suggests that rehearsal can function as a generative process in which subjects generate representations of possible probes and reduce response time by means of stimulus priming or activation. A similar view was expressed by William James (1890, p. 439) a long time ago.

A theoretical framework which is capable of capturing the salient findings of the generative processing studies is available in the LaBerge and Samuels (1974) model of reading. The model holds that visual information is extracted from the environment and transformed through a series of processing stages involving visual, phonological, and episodic memory codes until it reaches a semantic system where comprehension can occur. Of prime importance is the assumption that a stimulus code for a well-learned character may be activated in one of two ways: by the excitation of a particular set of visual features which are responding to the presence of that stimulus in the environment without the need of conscious attention (e.g., Eriksen \& Spencer, 1969; LaBerge \& Samuels, 1974; Posner \& Boies, 1971; Shiffrin \& Gardner, 1972), or through the momentary focus of attention by the person that stimulus's representa- 
tional code or codes in the absence of an external character.

The subject's rehearsal of the target characters in the studies of generative processes may be viewed as the shifting of attention from one item to another in the LaBerge and Samuels model. Since the characters were familiar English letters, attention is capable of activating the underlying codes for those letters as it shifts from one to another. Through activation or priming (Beller, 1971), attention can influence the perceptual encoding of a letter by increasing the rate of processing when the letter is presented (LaBerge \& Samuels, 1974). The priming through attention is seen as a preprocessing activity which influences probe pattern recognition processes by activating a particular letter's representation or processing pathway (Posner \& Boies, 1971) prior to external stimulation (Seamon \& Wright, 1976). Since generative processes are considered to influence pattern recognition, changing the task from manual classification to vocal identification should not alter the effect of generative processes on response time (Seamon, 1976b). Lastly, the encoding effect is confined to the last rehearsed item and is gone within $1 \mathrm{sec}$ because attention is directed to only one letter unit at any given time and it is forced to shift over the target representations at that rate due to the paced demands of the task (Seamon, 1976a).

An issue of obvious theoretical interest concerns the type of representational codes activated by generative processes. It is not known what representational codes were employed in the previously cited classification and identification experiments by Seamon and how generative processes affected those codes. It seems clear that subjects may employ different types of codes in a classification task (e.g., Clifton \& Tash, 1973; Posner, 1973; Sternberg, 1967) and that subjects may influence response time by generating visual representations of possible probe stimuli (Posner, Boies, Eichelman, \& Taylor, 1969; Tversky, 1969). But it is not clear whether the generated visual representations influenced encoding time or some other stage of information processing (Posner et al., 1969; Tversky, 1969), whether subjects could generate nonphysical representations which may affect encoding processes, and whether subjects could generate both visual and nonvisual representations in a task structured to encourage matching based on more than one coding level.

The present experiment examined the types of codes activated by generative processes in a character classification task. It was designed to permit responding on a nonvisual basis on some trials and a visual or nonvisual basis on other trials. Numerical stimuli were employed which could differ visually, yet have the same name and meaning (e.g., TWO, 2 , II, and . $)$. If subjects could classify stimuli only on the basis of a nonvisual code, encoding time should be reduced when the probe (e.g., 2) is visually the same as the last rehearsed target item (e.g., 2) or only phonologically or conceptually equal (e.g., II); there should be no effect on response time of the visual similarity of the probe and target items. Such a result would imply that generative processes can activate nonvisual representations.

Alternatively, if stimulus classification can be based on visual and nonvisual codes, encoding time should be facilitated in the same manner as before, but classifications based on visual identity should be faster over all conditions than those based on phonological or conceptual identity. These data would provide evidence for the use of visual and nonvisual representations in character classification (Posner, 1973), and, most importantly, they would indicate that generative processes may activate both visual and nonvisual memory codes prior to the encoding of an external stimulus.

\section{METHOD}

\section{Subjects}

The four subjects included the experimenter and three Wesleyan undergraduates who served as paid volunteers. All four subjects were highly practiced over multiple sessions in the character classification task prior to the experiment.

\section{Apparatus}

The apparatus and experimental environment were the same as those used previously (Seamon, 1976a; Seamon \& Wright, 1976).

\section{Procedure}

The experiment was conducted over nine sessions of approximately 168 trials each. The first session gave subjects familiarity with the different number forms. This session was considered practice and was not included in the final analysis.

In the basic character classification paradigm (Sternberg, 1967), a subject is presented with a short sequence of target characters followed by a blank interval and a probe. The task is to classify the probe in terms of target membership as quickly as possible. This basic paradigm was modified in two ways: first, subjects were asked to rehearse the target characters prior to the probe and report the last rehearsal after the probe (Seamon \& Wright, 1976); second, the rehearsal was externally paced by a recurring visual signal (Seamon, 1976a).

Over the eight experimental sessions, each subject was presented with 1,344 trials. There were 96 trials for Target Set Size 1, 384 trials for Target Set 2, and 864 trials for Target Set Size 3. Half of the correct responses in each target set size were positive and half were negative. For positive responses, all serial positions within a target array were equally probable. Trials in which subjects made an error were repeated at a randomly determined point

A trial consisted of an auditory beep, followed $.5 \mathrm{sec}$ later by the sequential presentation of the one, two, or three stimuli in the target array for $1 \mathrm{sec}$ each at a point in the top center of a GT:40 display screen. All items were displayed as bright green stimuli on a black background. The target stimuli were always one to three numbers presented sequentially in word form. The last target word was followed by a visual mask for $1 \mathrm{sec}$, which stopped the display of the last target item. The mask consisted of filling in each light point in the character spaces on the display 
screen formerly occupied by the target so that no character persistence remained. Depending upon target set size, a sequential series of 2 (Target Set Size 1), 3-4 (Target Set Size 2), or 4-6 (Target Set Size 3 ) recurring squares then appeared sequentially on the screen in the center of the position just occupied by the visual mask. Each square was shown for $1 \mathrm{sec}$ with its displayed intensity decremented in a stepwise fashion from bright to very dim over each $1-\mathrm{sec}$ interval. The recurring squares, which were presented in outline form only, served two purposes. First, since a probe item would be presented inside the square at some subsequent point in time, the square maintained the subject's fixation at the appropriate location on the display screen. Second, because the squares were recurring on a sequential basis, they served as an effective rehearsal pacer for the subject who was required to rehearse subvocally by visualizing and mouthing the target characters cyclically in the order shown at a rate of $1 \mathrm{sec} / \mathrm{character}$ in pace with the squares, e.g., the first target character was rehearsed with the presentation of the first square, and so forth. Dimming the brightness of the recurring square each time instead of simply re-presenting it for a short period of time within each second was thought to facilitate its use as a rehearsal pacer. No comparative data for different rehearsal pacer procedures are available.

The subjects were required to rehearse the target characters by visualizing and mouthing them subvocally in time with the square pacer in only one of the three number forms at any given time. Prior to each block of 28 trials, the subjects were given probability information on the likelihood of each number form as a probe (e.g., Arabic $=.5714$; Roman $=.2142 ; \mathrm{dot}=.2142)$. The subjects were told to rehearse the target numbers in the form of the high probability stimulus form only. Since subjects visualized the characters as part of their rehearsal, there was no difficulty in complying with the instruction. Within each block of 28 trials, the high-probability form was presented as a probe 16 times, and each of the two low-probability forms was used 6 times. Within each session, there were six blocks of 28 trials; each number form was used as the high-probability form in two blocks and as the low-probability form in four. Order of highprobability probe form within a session and between sessions was randomized. The stimulus forms used as targets and probes for both positive and negative responses were randomized from trial to trial and are shown in Table 1.

During the rehearsal sequence, after each target item had been rehearsed one time, a probe in either a high- or a low-probability form was presented inside the rehearsal pacer. Probes could be presented with the onset of the square rehearsal pacer or half way through the 1-sec period of square diminution. The intensity of the probe character did not vary and was the same as that used for the target set and the brightest period of a square exposure. Since a probe could be presented at either the onset of the square rehearsal pacer or half way through the 1-sec period of its diminution, there were two possible probe positions for each item that was rehearsed in a target. For Target Set Sizes 1, 2, and 3, there were thus two, four, and six possible probe positions for any given trial. Target numbers, probe form, response type, serial position for positive probes, rehearsal distance for positive probes, and probe position were all equally

Table 1

Target and Probe Stimulus Forms

\begin{tabular}{lccc}
\hline & \multicolumn{3}{c}{ Possible Probes } \\
\cline { 3 - 4 } Word & Arabic & Roman & Dot \\
\hline ONE & 1 & I & $:$ \\
TWO & 2 & II & $\vdots$ \\
THREE & 3 & III & $\vdots:$ \\
FOUR & 4 & IV & $::$ \\
FIVE & 5 & V & $\vdots:$ \\
SIX & 6 & VI & $\vdots$ \\
\hline
\end{tabular}

Table 2

Last Rehearsal Choices Over Target Set Size (TS), Item, and Probe Position

\begin{tabular}{rrrrrrrrr}
\hline & & \multicolumn{8}{c}{ Probe Position } \\
\cline { 3 - 10 } TS & Item & \multicolumn{1}{c}{1} & 2 & \multicolumn{1}{c}{ Rehearsal Pacer Square } \\
\hline 1 & A & 100.0 & 99.4 & & 4 & 5 & 6 & DKs \\
\hline & A & 66.1 & 24.3 & 26.5 & 75.5 & & & .3 \\
& B & 33.9 & 75.5 & 73.0 & 24.0 & & & .3 \\
& A & 67.7 & 23.9 & .5 & .2 & 25.2 & 74.8 & \\
3 & B & 31.5 & 75.5 & 71.2 & 25.0 & .2 & .4 & .1 \\
& C & .7 & .5 & 28.3 & 74.8 & 74.5 & 24.8 & \\
\hline
\end{tabular}

Note-Data are mean percentages averaged over four subjects. odd-numbered probe positions indicate probe onset; evennumbered positions indicate periods of diminution.

probable and randomly selected from trial-to-trial with the constraint that within any block of 28 trials, the selected probe forms must match the specified probe form probabilities given to the subject at the start of the block. Target set size was not equally probable, but was randomized over trials.

With the presentation of a probe character, the subject was required to indicate if it was a member of the preceding target by manually pressing either a yes or a no button with the left or right index finger, respectively. The probe, which remained on until a response was made, was classified as same if it had the same name and meaning as any of the target words; it did not have to have the same visual form as the rehearsal form. The subjects were encouraged to respond as quickly and as accurately as possible.

After manually classifying the probe, the subject was presented with a listing in multiple-choice format of all of the target characters from the array just shown along with an additional choice labeled "DON'T KNOW." The subject's task was to select the number of the alternative that corresponded to the item in the target array which was the last item rehearsed prior to the presentation of the probe. If the subject was unsure of the last rehearsal or otherwise unable to make an appropriate character selection, the subject was instructed to select the "DON'T KNOW" alternative. The responses to the multiple choice test were made manually via the subject's keyboard. A visual prompt instructed the subject to press the "DON'T KNOW" alternative if a selection had not been made within $5 \mathrm{sec}$. The rehearsal question was followed by a $2 \mathrm{sec}$ intertrial interval and the next trial.

\section{RESULTS AND DISCUSSION}

The percentage of last rehearsal choices as a function of target set size, item, and probe position is shown in Table 2. These data are means based on the percentage of last rehearsals for each subject. These data are strikingly similar to the last rehearsal patterns observed previously (Seamon, 1976a). For Target Set Size 1, subjects have only one item to rehearse, so this must be chosen as the last rehearsal at every probe position. With Target Set Sizes 2 and 3 , however, an opportunity for choice exists and a definite, cyclic rehearsal pattern emerges. Table 2 shows that subjects strongly anticipated each square cue by rehearsing the next character in the target sequence before its corresponding signal had ap- 
peared. This may readily be seen by looking at the status of the square rehearsal pacer at each probe position in Table 2 . The probe positions refer to the points in time that a probe could be presented; a probe could be presented inside the rehearsal square at either its onset (each odd-numbered probe position) or the period of diminution $1 / 2 \mathrm{sec}$ later (each even probe position). If subjects used the rehearsal pacer as intended, they would begin their rehearsal of an item on an odd probe position (square onset), maintain this item over the next even probe position (square diminution), and then shift their rehearsal to the next item in the target sequence at the next odd probe position (square onset again), etc., thereby rehearsing each character at a rate of $1 \mathrm{item} / \mathrm{sec}$. While the subjects did rehearse at a rate of 1 character/sec, they began each new rehearsal on the even probe positions (square diminution) instead of the next odd probe position (square onset). This is seen by a shift in the last rehearsal selection to the next item in the target on the even-numbered (square diminution) probe positions. These rehearsal patterns indicate that probe position alone cannot be used to specify the last rehearsed item; a last rehearsal identification is always necessary. Consistency between experiments is not evidence for the existence of only one rehearsal pattern. Informal observations have revealed that all people adopt a particular rehearsal pattern; some adopt a pattern like that observed in the present experiment, while others adopt a pattern that is consistent with the rehearsal pacer.

The mean reaction times based on the individual subject means collapsed over all conditions for the Arabic, Roman, and dot rehearsal forms were 588, 589 , and $582 \mathrm{msec}$, respectively. The comparable means for the Arabic, Roman, and dot probe forms were 558,608 , and $592 \mathrm{msec}$, respectively. These data indicate that there is little effect of rehearsal form per se on reaction time, but a large effect on probe form. The smaller mean time for Arabic probes relative to Roman or dot probes may be attributable to differences in preexperimental familiarity; most people have had more practice with Arabic number forms than with Roman or dot forms. An examination of latency by sessions and probe form showed this difference to be constant over all sessions; reaction times were higher in Sessions 1 and 2 than in all subsequent sessions, but the ordering of the probe forms remained consistent throughout.

Since response times varied for the different probe forms, it was necessary to normalize the data to examine the mean reaction times for each rehearsal form and probe form. The data were normalized by subtracting the appropriate row or column means and adding the grand mean for each rehearsal form and probe form separately; they are shown in Table 3.
The results very clearly show reaction time to be faster when the probe form matches the rehearsal form than when it differs. If the probe form and rehearsal form differ, the form of the probe does not matter. These data strongly suggest that subjects followed the instructions of rehearsing only the highprobability form of the probe; there is no evidence to indicate that all three forms were generated with each rehearsal. An analysis of error percentages by rehearsal form and response type showed no effect of a speed-accuracy tradeoff. Errors did not vary with Rehearsal Form or Response Type (both Fs $<1.0$ ).

To obtain more observations per data point and reduce the number of data points, the mean reaction times for each rehearsal form and probe form were reduced to two conditions: a high-probability probe (rehearsal form and probe form were the same) and a low-probability probe (rehearsal form and probe form were different). Mean latencies were obtained for each subject and averaged over subjects for correct responses for each target set size, probe probability, response type, and rehearsal distance function. The rehearsal distance functions were defined (Seamon, 1976a, 1976b; Seamon \& Wright, 1976) as the computed circular distance between the probe character an the last character rehearsed, such that the distance (D) is 0 when these characters are the same (either visually, phonologically, or conceptually), 1 when the probe is the character rehearsed just previous to the last rehearsed item, and so on. Target Set Sizes 1, 2, and 3 may have rehearsal distance values of 0,0 or 1 , and 0,1 , or 2 , respectively. The mean reaction times and average standard errors over four subjects for each condition are shown in Figure 1. The general findings may be summarized by noting that (1) reaction time increased monotonically over target set size, (2) positive responses were faster than negative resonses, (3) there were significant and large effects of probe form probability on positive responses, but little effect on negative responses, and (4) reaction times for positive responses were faster for $D=0$ than for $D>0$ when the probe was visually the same as the last rehearsed target item or only phonologically or conceptually the

Table 3

Normalized Mean Reaction Times for Each Rehearsal Form and Probe Form

\begin{tabular}{lccc}
\hline & \multicolumn{3}{c}{ Probe Form } \\
\cline { 2 - 4 } $\begin{array}{l}\text { Rehearsal } \\
\text { Form }\end{array}$ & Arabic & Roman & Dot \\
\hline Arabic & 565 & 597 & 598 \\
Roman & 595 & 568 & 595 \\
Dot & 599 & 594 & 566 \\
\hline
\end{tabular}

Note-Data are means expressed in milliseconds. 


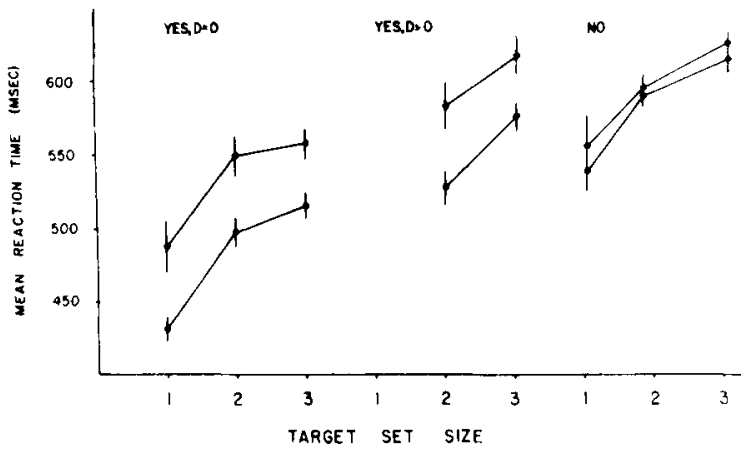

Figure 1. Mean reaction time plus or minus one average standard error for each target set size, response type, and rehearsal distance function. High- and low-probability probe conditions are represented by the upper and lower functions for Yes, D = 0; Yes, D > 0; and No, respectively. For Target Set Size 3, the rehearsal distance functions for $D=1$ and $D=2$ were averaged to simplify viewing.

same. These data provide strong support for the hypotheses that (1) subjects classified stimuli on the basis of visual and nonvisual representations by virtue of the difference between reaction times for highprobability and low-probability probe forms, and (2) rehearsal may function as a generative process to activate both visual and nonvisual memory codes by virtue of the difference between $D=0$ and $D>0$ for both high- and low-probability probe forms. While these data might suggest that subjects may activate visual and nonvisual codes at the same moment in time, the results do not force this conclusion. If generative processes produce effects which persist in time, albeit briefly, then it is possible for visual and nonvisual codes to be activated separately, yet overlap in time. The present data cannot differentiate these possibilities.

The manner in which generative processes interact with classification processes to produce the results found in Figure 1 can be explained by a coding model which assumes that subjects can classify stimuli on a visual or nonvisual basis in different conditions. When the subjects are rehearsing in a high-probability form and the probe is presented in a low-probability form, all classifications must be based on a nonvisual attribute, such as stimulus name, and the probe must be encoded to the appropriate level of analysis. Generative processes may reduce the time needed to achieve a nonvisual level of probe encoding by activating a nonvisual representation of the probe during the last rehearsal. For high-probability probe conditions, the situation is more complex. When the rehearsal and probe form are the same, classification for positive responses could be based on both visual and nonvisual attributes, while those for negative responses would require a nonvisual attribute. Since the response rule requires a positive decision for a probe that is the same in appearance, name, or meaning as a target item, a match on the basis of appearance is sufficient for a positive response, but a match failure on this basis is not sufficient for a negative response. If, as Posner (1973) has demonstrated, a probe can be encoded automatically to several levels and encoding to a physical levei is completed sooner than to a nonphysical level, then the results of Figure 1 may be described in terms of coding processes and generative processes. First, positive responses for the high-probability conditions are faster than the corresponding low-probability conditions, because the former are based on visual matches and probe encoding to a visual level is faster than encoding to a nonvisual level. Second, generative processes may activate a visual representation of the probe during the last rehearsal and facilitate the encoding of a probe to a visual level. Generative processes may always activate underlying visual and nonvisual codes, but the manner in which encoding time is reduced will depend upon the level of probe encoding necessary and the appropriateness of the codes activated. Generating an incorrect number form, for example, will not be detrimental to performance if the appropriate number name has been activated and the task requires encoding of the probe to the name level of analysis.

\section{Odd and Even Probe Positions}

Seamon (1976a) found differences in the effects of generative processes on response time when odd (rehearsal pacer onset) and even (rehearsal pacer diminution) probe positions were examined separately. At even probe positions, generative processes affected probe encoding time only if the probe matched the last rehearsal $(D=0)$; no other target items were affected. These data replicated the results of the self-paced studies (Seamon, 1976b; Seamon \& Wright, 1976). At odd probe positions, however, different results were observed. One subject showed evidence of a spreading effect of generative processes on items other than the last rehearsal, while the other subject showed no effect of generative processes on any target items (Seamon, 1976a). These data might suggest that generative processes may be timeextended processes which yield different results when examined at different points in time. Figure 2 presents mean reaction times and average standard errors (see figure caption) for each response type, rehearsal distance function, odd or even probe position, and high or low probe probability over target set size. The main observations may be summarized as follows: There is an effect of rehearsal on reaction time for high- and low-probability probe forms, i.e., the probe form does not have to visually match the rehearsal form as faster reaction times were observed for $D=0$ under all conditions; the effect of rehearsal 


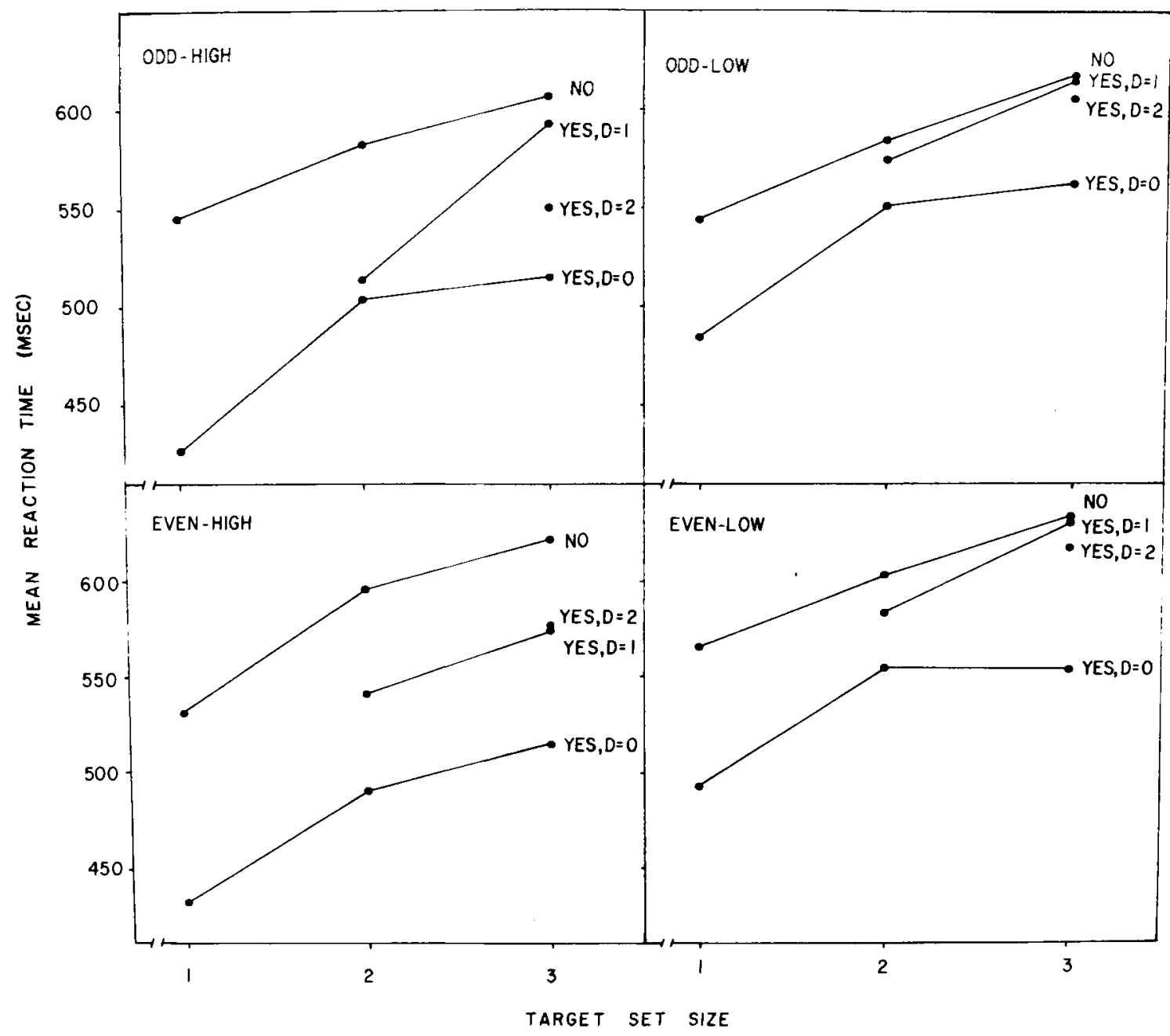

Figure 2. Mean reaction time for each target set size, response type, rehearsal distance function, odd or even probe position, and high- and low-probability probe form. Average standard errors were equal for odd and even probe positions within high $(13,13$, and $12 \mathrm{msec}, \mathrm{D}=0$, Set Size 1-3; 16 and $15 \mathrm{msec}, \mathrm{D}=1$, Set Size 2.3; $13 \mathrm{msec}, \mathrm{D}=2$, Set Size 3, 19, 11, and $8 \mathrm{msec}$; No, Set Size 1-3) or low $(26,18$, and $15 \mathrm{msec}, \mathrm{D}=0$, Set Size 1-3; 24 and $19 \mathrm{msec}$, $D=1$, Set Size 2-3; $19 \mathrm{msec}, \mathrm{D}=2$, Set Size 3;27, 13, and $9 \mathrm{msec}$, No, Set Size 1-3) probability probe forms.

on response time is confined to the last rehearsed item on even probe positions, but can show diminished effects on the next item to be rehearsed as well as on odd probe positions. The latter observation is seen most clearly in the odd and even probe position data for high-probability probe forms. There is an effect of rehearsal at both odd and even probe positions, but only on even probe positions is the effect confined to the last rehearsed item $(D=0)$. At odd probe positions, there is a large effect of rehearsal on the last rehearsed item $(D=0)$ and a smaller effect on the target item to be rehearsed just after the last rehearsal $(D=1$ in Target Set Size 2 and $\mathrm{D}=2$ in Target Set Size 3). Assuming that rehearsal activates number representations in a cyclic fashion, it is as if the effect of the next number activation has begun at the odd probe positions, and, while it is not yet as strong as the currently rehearsed item $(D=0)$, it can have some effect on reaction time. At even probe positions, $.5 \mathrm{sec}$ later, the activation for the to-be-rehearsed item grows, while that of the last rehearsed item dissipates to its baseline level. At this point, there is a new last rehearsal choice and the reaction time functions for negative responses and positive responses at each rehearsal distance are virtually parallel over target set size. The lack of comparable differences over probe position for lowprobability probe forms is not clear, although these data are, by necessity, based on fewer observations. ${ }^{1}$

The finding that rehearsal reduced response times for low-probability probes as well as high-probability probes, together with the finding that high-probability probes yielded shorter latencies than low-probability probes provides strong support for the hypothesis 
that rehearsal functions as a generative process to influence probe encoding by activating visual and nonvisual codes. A comparison of the high- and lowprobability probe data in Figure 2 shows that reaction time increased over target set size at comparable rates in both conditions. This indicates that a translation process (e.g., Clifton, Sorce, \& Cruse, 1977) was not present in these data and suggests that subjects could scan the character representations in shortterm memory on either a visual or nonvisual basis at comparable speeds. Table 4 presents error percentages as a function of experimental conditions. As before, there is no evidence to suggest a speedaccuracy tradeoff interpretation of these data. Errors tend to be higher at odd than at even probe positions, to increase over target set size, and, most importantly, to be greater for target items that are different from the last rehearsal. Generative processes have consistently shown comparable effects on speed and accuracy.

An examination of the mean reaction time difference between $D>0$ and $D=0$ for each rehearsal form and probe form in Target Set 3 is shown in Table 5. The results, based on even probe positions where Ds $>0$ were equal, show the amount of latency saved when the probe is the same as the last rehearsal $(D=0)$ as opposed to different from the last rehearsal $(\mathrm{D}>0)$ to be roughly comparable across all conditions. Similar effects were found for Target Set 2, although there was more variability due to fewer observations. Clearly, there is a reduction in reaction time when the probe has the same name and meaning as the last rehearsal, regardless of the physical appearance of each.

\section{Additivity Tests}

Assuming that character classification is composed of probe encoding, memory comparison, binary decision, and response organization stages (Sternberg, 1969), the additive factors method may be employed

Table 4

Mean Percent Errors by Target Set Size and Response Condition

\begin{tabular}{|c|c|c|c|c|}
\hline \multirow{2}{*}{$\begin{array}{l}\text { Response } \\
\text { Condition }\end{array}$} & \multirow{2}{*}{$\begin{array}{l}\text { Probe } \\
\text { Position }\end{array}$} & \multicolumn{3}{|c|}{ Target Set Size } \\
\hline & & 1 & 2 & 3 \\
\hline No & $\begin{array}{l}\text { Odd } \\
\text { Even }\end{array}$ & $\begin{array}{l}7.8 \\
6.9\end{array}$ & $\begin{array}{l}7.1 \\
2.7\end{array}$ & $\begin{array}{l}6.4 \\
2.8\end{array}$ \\
\hline $\begin{array}{l}\text { Yes, } \\
D=0\end{array}$ & $\begin{array}{l}\text { Odd } \\
\text { Even }\end{array}$ & $\begin{array}{l}1.3 \\
0.0\end{array}$ & $\begin{array}{r}3.5 \\
.5\end{array}$ & $\begin{array}{l}2.5 \\
3.1\end{array}$ \\
\hline $\begin{array}{l}\text { Yes, } \\
D=1\end{array}$ & $\begin{array}{l}\text { Odd } \\
\text { Even }\end{array}$ & & $\begin{array}{l}4.7 \\
4.0\end{array}$ & $\begin{array}{r}10.2 \\
6.4\end{array}$ \\
\hline $\begin{array}{l}\text { Yes, } \\
\mathrm{D}=2\end{array}$ & $\begin{array}{l}\text { Odd } \\
\text { Even }\end{array}$ & & & $\begin{array}{l}4.4 \\
5.0\end{array}$ \\
\hline
\end{tabular}

Note-Data are percentages.
Table 5

Magnitude of Generative Processes Effect as a Function of Rehearsal Mode and Probe Form

\begin{tabular}{cccc}
\hline \multirow{2}{*}{$\begin{array}{c}\text { Rehearsal } \\
\text { Mode }\end{array}$} & Arabic & Roman & Dot \\
\cline { 2 - 4 } & 60 & 82 & 79 \\
Arabic & 75 & 62 & 78 \\
Roman & 57 & 41 & 67 \\
Dot & &
\end{tabular}

Note-Data are mean differences between $D>0$ and $D=0$ for Set Size 3 expressed in milliseconds.

to determine the locus or loci of different independent variables on the processing sequence (Sternberg, 1969). Previous work by Sternberg (1969) has indicated that memory set size affects the comparison process, while type of response influences the binary decision stage. The findings that generative processes, as indexed by rehearsal distance, influence reaction time in a character identification task, where a comparison stage is logically absent (Seamon, 1976b), and do not interact with memory set size in classification tasks (Seamon, 1976b; Seamon \& Wright, 1976; present experiment) indicate that generative processes are not affecting the comparison stage. The fact that generative processes interact with target set size at odd probe positions in the present experiment is attributable to the view that more than one representation may be activated simultaneously at these positions.

While it has been argued previously that generative processes affect primarily probe encoding processes (Seamon, 1976b), a more direct test of this hypothesis is provided by tests of additivity. Figure 3 presents mean reaction times for high- and low-probability probe forms as a function of rehearsal distance, memory set size, and response type. The means are based on the even probe positions, since more than one representation might be activated at the odd positions. Analyses, however, were based on both even probe positions and all probe positions, and any discrepancy will be noted. It is evident in Part $\mathbf{A}$ of Figure 3 that probe form probability does not interact with generative processes. An analysis of variance of positive responses by rehearsed distance, memory set size, and probe form probability found nonsignificant interactions of Rehearsal. Distance by Probe Probability, $\mathrm{F}<1.0$ (Figure 3A), Rehearsal Distance by Set Size, $F(1,3)=4.79, p>.05$, Set Size by Probe Probability, $F(1,3)=2.00, \mathrm{p}>.05$ (Figure 3B), and Rehearsal Distance by Set Size by Probe Probability, $F(1,3)=1.35, p>.05$. For analyses based on all probe positions, only the Rehearsal Distance by Set Size interaction was significant, $\mathrm{F}(1,3)=12.24, \mathrm{p}<.05$. This is not surprising 


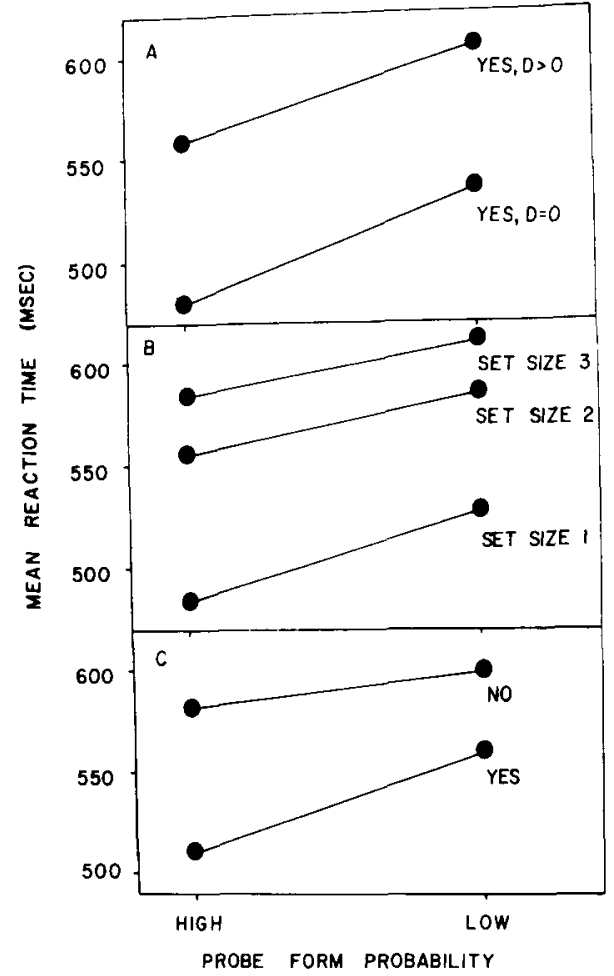

Figure 3. Mean reaction times for high- and low-probability probe forms by rehearsal distance, target set size, and response type.

because, as was noted earlier in the discussion of Figure 2, differential effects of rehearsal distance over set size were found for the odd probe positions, suggesting that more than one representation might be activated. These data indicate that for even probe positions, generative processes, memory set size, and probe form probability are affecting different processing stages.

Similar analyses of latencies by response type, memory set size, and probe probability reveal significant interactions of Response Type by Probe Probability, $F(1,3)=10.20, p<.05$ (Figure $3 C$ ), Response Type by Set Size, $F(2,6)=10.10, p<.05$, and Set Size by Probe Probability, $F(2,6)=7.24$, $\mathrm{p}<.05$. The Response Type by Set Size interaction is unimportant, as it merely reflects the presence of generative processes in the positive responses. This interaction has always been found when positive responses have not been analyzed in terms of rehearsal distance (e.g., Seamon \& Wright, 1976). However, the other interactions are of great importance as they serve to localize the effect of probe form probability primarily in the binary decision stage. ${ }^{2}$ While this factor may affect the comparison stage as well when negative responses are included, it is clear that probe form probability is not affecting either the probe encoding or response organization stages. By elimination, generative processes must affect one or both of these stages. Instead of thinking of generative processes in dichotomous terms, it may be more appropriate to consider that rehearsal distance may affect both probe encoding and response organization; specific conditions may determine under what circumstances each is affected (cf. Biederman \& Stacy, 1974; Egeth \& Bevan, 1973; Kornblum, 1973; Pachella, 1975). One way of determining the magnitude of the encoding and response effects is to use the subtraction method employed by Eichelman (1970), Hinrichs and Krainz (1970), and LaBerge, LeGrand, and Hobbie (1969), among others (see Kornblum, 1973), to examine separately the effects of stimulus and response repetition. The same procedures could be applied to the present data by noting that for Yes, $D=0$ responses, the subject had a stimulus and response repetition, for Yes, D $>0$ responses, the subject had only a response repetition, while for No responses, the subject had neither form of repetition. ${ }^{3}$ If it is assumed that the activation of a stimulus code also activates its associated response (LaBerge \& Samuels, 1974), then it is reasonable to attribute the difference between $\mathrm{D}>0$ and $\mathrm{D}=0$ to the effects of generative processes on probe encoding (stimulus expectancy) and the difference between No and D $>0$ to the effects on response organization (response expectancy).

The above procedure was used to obtain difference scores for Set Sizes 2 and 3, with the scores then averaged over set size. Obviously, the difference scores would have meaning only if generative processes affected one item at a time. For this reason, only data from the even probe positions were considered. The results show latency differences attributable to probe encoding and response organization of 52 and $33 \mathrm{msec}$, respectively. The results from an earlier externally paced rehearsal experiment (Seamon, 1976a) show comparable results of 64 and $29 \mathrm{msec}$, respectively. These results, while offered tentatively, are consistent with the view of perceptual expectancy (Egeth \& Bevan, 1973; Pachella, 1975) and repetition effects (Kornblum, 1973) cited previously, and they are not contradicted by the additivity tests on the present data.

Finally, a minor note of some theoretical interest concerns the effect of serial position of positive probes on reaction time. The most salient feature of these data is the finding of a strong primacy effect. This effect has been repeatedly observed (Seamon, 1976a; Seamon \& Wright, 1976) and cannot be attributed to either generative processes or differences in rehearsal frequency (cf. Cavanagh, 1976) as the means were equated on these factors. The observed primacy effects may reflect differences in general preparation (cf. Posner \& Boies, 1971) as the subjects may rhythmically stress or accentuate the first member as they cyclically rehearse the target items. 


\section{GENERAL DISCUSSION}

The results from the present experiment may be summarized as follows: Subjects are able to rehearse a target set of one to three characters in time with an external pacer; a particular rehearsal pattern emerges when there is more than one item to rehearse such that subjects tend to anticipate the next rehearsal cue; rehearsal affects reaction time differently at odd and even probe positions; reaction time is reduced only when the probe matches the last rehearsed item on even probe positions, while it is reduced for the last rehearsed item and, to a lesser extent, the next to-be-rehearsed item on odd probe positions; the duration of the effect of rehearsal on reaction time is short-lived, lasting at most for $1 \mathrm{sec}$ in the present conditions; comparable rehearsal effects occur for characters that are visually the same or only phonologically and conceptually the same as the last rehearsed item.

One way to account for the last rehearsal choices and the latency data is to assume that rehearsal functions as a time-extended generative process. These effects may appear to be discrete, all-or-none phenomena when gross methods are employed (Seamon, 1976b; Seamon \& Wright, 1976), but more refined techniques may alter that view. Such techniques as externally paced rehearsal and precise temporal positioning of probes in relation to the rehearsal suggest that generative processes operate in a continuous, rather than discrete, fashion; their effects are better captured by the idea of a distribution of activation that builds and then declines over time with each rehearsal. Figure 4 shows how these ideas might apply to account for the present data. Hypothetical activation distributions are shown separately for Target Set Sizes 2 and 3 along with the observed rehearsal patterns and rehearsal distance functions at each possible probe position. It is assumed that
(1) rehearsing a target character activates its internal representation, (2) activation can be represented by a continuous distribution, (3) the reaction time to a probe is an inverse function of the degree to which its internal representation has been activated, and (4) the last rehearsal choice is based on the internal representation that currently has the greatest degree of activation.

The cyclic nature of rehearsal activation is analogous to the activity present in mental rotation (Shepard, 1975). As Cooper has stated, "The internal process passes through a series of states at each one of which the subject is especially prepared for the presentation of a particular external object" (Cooper, 1976 , p. 296). The generative processing studies would appear to be similar; instead of cyclically rotating a single object, subjects cyclically activate a series of object representations.

Latencies are comparable at odd and even probe positions for $\mathrm{D}=0$, because a representation may be maintained at high activation for at least $.5 \mathrm{sec}$ (e.g., the B function at Probe Positions 2 and 3 in Figure 4). Differences are obtained when probing the to-be-rehearsed item at odd and even probe positions because at odd probe positions the next item has already begun to receive enough activation buildup to influence reaction time, but not enough to alter the last rehearsal choice (e.g., the A function at Probe Position 3 in Figure 4); at even probe positions, $.5 \mathrm{sec}$ later, the buildup in activation for that item is so great and the decay of activation from the preceding item so fast, that that item is now chosen as the last rehearsal, and it alone, for the moment, can affect reaction time (e.g., the $\mathrm{A}$ function at Probe Position 4 in Figure 4). ${ }^{4}$ The entire effect of buildup and decay would appear to last in the order of $1-2 \mathrm{sec}$, although it is not clear if this reflects the actual duration of this process or the duration due to the paced demands of the task. Additional
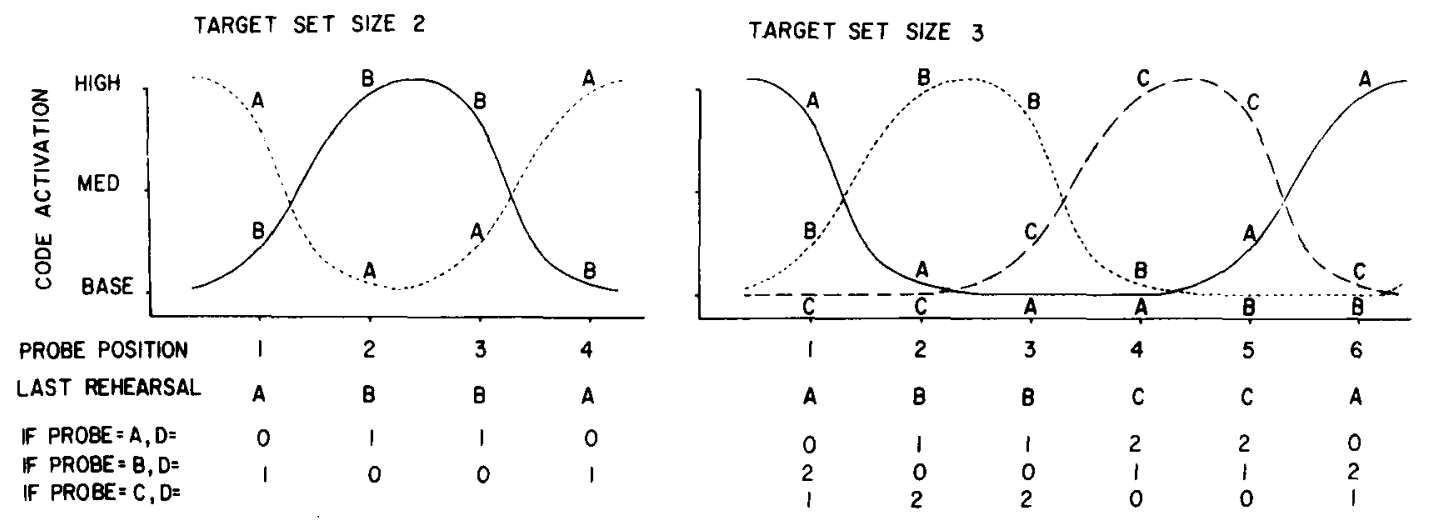

Figure 4. Hypothetical activation distributions, rehearsal patterns, and rehearsal distance functions for Target Set Sizes 2 and 3. 
information could be obtained on this point by varying the rate of rehearsal and observing the effect on the duration and magnitude of generative processes.

In terms of the issue stated originally, it seems apparent that generative processes affect probe encoding by activating both visual and nonvisual memory representations for well-learned stimuli. The activated representations serve to increase the rate of processing (LaBerge \& Samuels, 1974) of those items to their appropriate levels of analysis for character classification.

\section{REFERENCES}

BeLler, H. K. Priming: Effects of advance information on matching. Journal of Experimental Psychology, 1971, 87, 176-182.

Biederman. I., \& Stacy, E. W., JR. Stimulus probability and stimulus set size in memory scanning. Journal of Experimental Psychology, 1974, 102, 1100-1107.

Boring, E. G. A history of experimental psychology (2nd ed.). New York: Appleton-Century-Crofts, 1950.

Cavanagh, P. Holographic and trace strength models of rehearsal effects in the item recognition task. Memory \& Cognition, 1976, 4, 186-199.

Clifton, C., JR., Sorce, P., \& Cruse, D. The translation effect in memory search. Cognitive Psychology, 1977, 9, 1-30.

Clifton, C. E., JR., \& Tash, J. Effect of syllabic word length on memory-search rate. Journal of Experimental Psychology, 1973, 99, 231-235.

COOPER, L. A. Demonstration of a mental analog of an external rotation. Perception \& Psychophysics, 1976, 19, 296-302.

Egeth, H., \& BevaN, W. Attention. In B. Wolman (Ed.), Handbook of psychology. New York: Prentice-Hall, 1973.

Eichelman, W. H. Stimulus and response repetition effects for naming letters at two response-stimulus intervals. Perception \& Psychophysics, 1970, 7, 94-96.

Eriksen, C. W., \& Spencer, T. Rate of information processing in visual perception: Some results and methodological considerations. Journal of Experimental Psychology Monograph, 1969, 72, No. 2, Part 2.

Hinrichs, J. V., \& Krainz, P. L. Expectancy in choice reaction time: Anticipation of stimulus or response? Journal of Experimental Psychology, 1970, 85, 330-334.

JAMES, W. The principles of psychology. New York: Dover, 1950. (Originally published in 1890.)

Kornblum, S. Sequential effects in choice reaction time: A tutorial review. In S. Kornblum (Ed.), Attention and performance IV. Nw York: Academic Press, 1973.

KüLPE, O. Outlines of psychology. New York: Macmillan, 1895.

LaBerge, D., LeGrand, R., \& Hobmie, R. K. Functional identification of perceptual and response biases in choice reaction time. Journal of Experimental Psychology, 1969, 79, 295-299.

LABerge, D., \& SAmuels, S. J. Toward a theory of automatic information processing in reading. Cognitive Psychology, 1974, 6. 292-323.

Pachella, R. G. The effect of set on the tachistoscopic recognition of pictures, In P. M. A. Rabbitt \& S. Dornic (Eds.), Attention and performance V. New York: Academic Press, 1975.

Posner, M. I. Coordination of codes. In W. G. Chase (Ed.), Visual information processing. New York: Academic Press, 1973.
Posner. M. I., \& BoIEs, S. J. Components of attention. Psychological Review, 1971, 78, 341-408.

Posner, M. I., Boies, S. J., Eichelman, W. H., \& Taylor, R. L. Retention of visual and name codes of single letters. Journal of Experimental Psychology Monograph, 1969, 79. No. 1, Part 2.

Seamon, J, G. Generative processes in character classification: II. A refined testing procedure. Bulletin of the Psychonomic Society, 1976, 7, 327-330. (a)

Seamon, J. G. Effects of generative processes on probe identification time. Memory \& Cognition, 1976, 4, 759-762. (b)

Seamon, J. G., \& Wrughr, C. E. Generative processes in character classification: Evidence for a probe encoding set. Memory \& Cognition, 1976, 4, 96-102.

SHEPARD, R. N. Form, formation, and transformation of internal representations. In R. Solso (Ed.), Information processing and cognition: The Loyola Symposium. Hillsdale, N.J: Erlbaum. 1975.

Shiffrin, R. M., \& Gardner, G. T. Visual processing capacity and attentional control. Journal of Experimental Psychology, $1972,93,72-82$.

Sterne ERG, S. Two operations in character recognition: Some evidence from reaction time measurements. Perception \& Psychophysics, 1967, 2, 45-53.

STERNBERG, $S$. The discovery of processing stages: Extensions of Donder's method. In W. G. Koster (Ed.), Attention and performance II. Amsterdam: North Holland, 1969. Pp. 276-315.

TITCHENER, E. B. Lectures on the elementary psychology of feeling and attention. New York: Macmillan, 1908.

TVERSKY, B. Pictorial and verbal encoding in a short-term memory task. Perception \& Psychophysics, 1969, 6, 225-233.

\section{NOTES}

1. Results identical to the high-probability probe data were found in an unpublished study which presented probes only in the same form as the target stimuli. This consistency across experiments indicates that the different effects of generative processes for odd and even probe positions is reliable.

2. A similar interaction of Probe Form by Response Type was found in unpublished data noted in Seamon and Wright (1976). Reaction times for uppercase probes were much faster than for lowercase probes for positive responses than for negative responses at each set size. In this study, probe form probabilities were equal (.5).

3. For the sake of simplicity, Yes, $D=0$, high-probability and low-probability responses are both labeled stimulus repetitions, since the last rehearsal and the probe have the same name and meaning.

4. It might be argued that at odd probe positions there is confusion among the last rehearsal choices in Target Set Sizes 2 and 3 , such that $D=0$ rehearsals are mislabeled by the subjects as $D>0$. This would have the effect of lowering certain $D>0$ latencies. This hypothesis predicts that uncertainty should be reflected in the last rehearsal choices between items; the frequency with which one item is selected over another should be lower for odd than for even probe positions. Three of the four subjects show no difference in the frequency of last rehearsal choices at odd or even probe positions, and the latency data from these subjects show the same pattern of results as shown in Figure 2. Confusion among last rehearsals does not appear to account for the data.

(Received for publication September 26, 1977; revision accepted March 14,1978 .) 